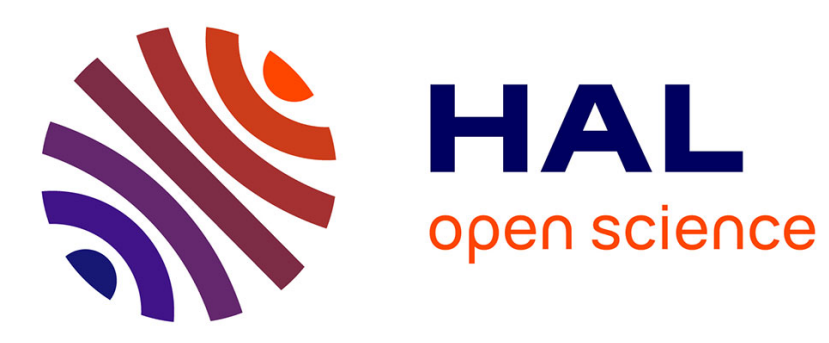

\title{
A hierarchical variational Bayesian approximation approach in acoustic imaging
}

Ning Chu, Ali Mohammad-Djafari, Nicolas Gac, José Picheral

\section{To cite this version:}

Ning Chu, Ali Mohammad-Djafari, Nicolas Gac, José Picheral. A hierarchical variational Bayesian approximation approach in acoustic imaging. 34th International Workshop on Bayesian Inference and Maximun Entropy Methods in Science and Engineering (MaxEnt'14), Sep 2014, Amboise, France. pp.572 - 579, 10.1063/1.4906024 . hal-01103784

\section{HAL Id: hal-01103784 \\ https://hal-centralesupelec.archives-ouvertes.fr/hal-01103784}

Submitted on 15 Jan 2015

HAL is a multi-disciplinary open access archive for the deposit and dissemination of scientific research documents, whether they are published or not. The documents may come from teaching and research institutions in France or abroad, or from public or private research centers.
L'archive ouverte pluridisciplinaire HAL, est destinée au dépôt et à la diffusion de documents scientifiques de niveau recherche, publiés ou non, émanant des établissements d'enseignement et de recherche français ou étrangers, des laboratoires publics ou privés. 


\title{
A Hierarchical Variational Bayesian Approximation Approach in Acoustic Imaging
}

\author{
Ning $\mathrm{Chu}^{*, \dagger}$, Ali Mohammad-Djafari*, Nicolas Gac* and José Picheral ${ }^{* *}$ \\ *Laboratoire des signaux et systèmes (L2S), CNRS-SUPELEC-UNIV PARIS-SUD, France \\ ${ }^{\dagger}$ College of Electrical Science and Engineering, National University of Defense Technology, China \\ ${ }^{* *}$ Dept. Signal et Systèmes Électroniques, École Supérieure d'Électricité (SUPELEC), France
}

\begin{abstract}
Acoustic imaging is a powerful technique for acoustic source localization and power reconstruction from limited noisy measurements at microphone sensors. But it inevitably confronts a very ill-posed inverse problem which causes unexpected solution uncertainty. Recently, the Bayesian inference methods using sparse priors have been effectively investigated. In this paper, we propose to use a hierarchical variational Bayesian approximation for robust acoustic imaging. And we explore the Student-t priors with heavy tails to enforce source sparsity, and to model nonGaussian noise respectively. Compared to conventional methods, the proposed approach can achieve the higher spatial resolution and wider dynamic range of source powers for real data from automobile wind tunnel.
\end{abstract}

Keywords: Acoustic imaging; Variational Bayesian Approximation; Student-t prior; NonGaussian noise

PACS: 43.60.+d, 02.50.Cw, 07.05.Pj

\section{INTRODUCTION}

Nowadays, acoustic imaging methods play more and more important roles in industry which have to take the (aero)acoustics performance into account, such as vehicle design, noise control and wind energy generation etc. In general, the conventional Beamforming method [1] can give a direct and fast acoustic power imaging, but its spatial resolution is often very coarse at low frequencies. Based on the beamforming, the forward model of acoustic power propagation can be modeled by a determined linear system of equations in frequency domain [2]: $\mathbf{y}=\mathbf{C x}$, where $\mathbf{x} \in \mathbb{R}^{N \times 1}$ denote the unknown acoustic power vector on the source plane consisted of $N$ identical patches; $\mathbf{y} \in \mathbb{R}^{N \times 1}$ denote the observed beamforming power vector at microphone array; $\mathbf{C} \in \mathbb{R}^{N \times N}$ denote the source power propagation matrix, which not only depends on the geometric distance between the source and sensor, but also relies on the array geometry for a given frequency. The matrix $\mathbf{C}$ is usually a shift-variant and singular matrix, thus the problem of solving $\mathbf{y}=$ $\mathbf{C x}$ will be ill-posed. Recently, the Deconvolution Approach for Mapping of Acoustic Source (DAMAS) method [2] has been effectively applied in wind tunnel experiments by NASA. For super resolution imaging under strong Gaussian noise, many methods with sparsity constraints have been extensively developed [3]. However, the sparsity parameters have to be selected empirically. In order to obtain the robust parameter estimations, the Bayesian inference approaches with the sparsity favoring priors have been widely investigated $[5,6,7,8]$. We propose to use the Double Exponential (DE) prior, and apply the joint Maximum A Posteriori (MAP) estimation [9] to improve upon 
the DAMAS and its extensions. However, the proposed joint MAP method often suffers from the time-consuming non-quadratic optimization.

In this paper, our motivation aims to obtain the robust acoustic imaging on the vehicle surface in wind tunnel tests, which can be effectively applied in car manufacture. To overcome the limitations of joint MAP, we propose to use the hierarchical Bayesian inference via Variational Bayesian Approximation (VBA). Moreover, Student-t priors are used to not only enforce the sparsity of source power distribution, but also to model the non-Gaussian distributed noise.

This paper is organized as follows: we first introduce the forward model of acoustic power propagation. The proposed VBA approach is then discussed in detail and used to solve the forward model. Subsequently, the method validation is carried out on simulations and real data respectively, and followed by our conclusions.

\section{PROBLEM STATEMENT}

We consider $K$ unknown sources on the source plane and $M$ number of microphone sensors located on a non-uniform 2D array. Before modeling, some assumptions are made: The acoustic sources are uncorrelated, monopoles [2]; Microphone sensors are omni-directional with unitary gain; In addition, complex reverberations in open wind tunnel are ignored.

After discretizing the source plane into $N$ identical patches $(N>>K)$, we get $N$ potential sources within which only $K$ real sources are present non-zero items. So that it is beneficial to consider this as a sparse-vector of length $N$ with $\mathrm{K}$ components. We should note that background noise in wind tunnel is mainly composed by the noise at the sensors, and the model uncertainty [9] caused by acoustic multi-path propagations such as reflection and refraction. In this case, the background noise should not be modeled by the ideal Gaussian white spectrum [2,9], but by non-Gaussian colored noise. Therefore we propose the forward model of acoustic power propagation in colored noise as:

$$
\mathbf{y}=\mathbf{C x}+\xi,
$$

where $\xi$ denote the colored noise vector; the propagation matrix $\mathbf{C}=\left\{\left\|\mathbf{a}_{i}^{H} \mathbf{a}_{j}\right\|_{2}^{2} /\left\|\mathbf{a}_{i}\right\|_{2}^{2}\right\}_{N \times N}$ is derived in [9], where the beamforming steering vector $\mathbf{a}_{i}=\left\{\frac{\exp \left[-j 2 \pi f r_{i, m}\right] / c_{0}}{r_{i, m}}\right\}_{M \times 1}$ [2] depends on the geometric distance $r_{i, m}$ between source $i$ and sensor $m$ at a given frequency $f$, with $c_{0}$ being the acoustic speed in the air. Thus we can see that (1) is a linear determined system of equations to solve $\mathbf{x}$ from the given $\mathbf{y}$ and distortions $\xi$.

\section{PROPOSED VBA INFERENCE APPROACH}

For the inverse problem in (1), some prior knowledge or necessary constraints on source powers $\mathbf{x}$ and colored noise $\xi$ should be investigated in order to reduce the uncertainty of solutions. Let $\mathbf{y}$ denote the observed data, $\theta$ the unknown parameters. The inverse problem regularized based on priors can be solved by the following Bayesian inference approaches $[5,6]$ : If we assign the specific prior probability $p(\xi)$ to noise vector $\xi$, we can 
define the likelihood $p(\mathbf{y} \mid \mathbf{x}, \theta)$, namely $p(\xi)=p(\mathbf{y}-\mathbf{x} \mid \theta)$, which can be solved by the classical Maximum Likelihood (ML) estimation as $(\hat{\mathbf{x}}, \hat{\theta})_{M L}=\arg \max _{\mathbf{x}, \theta}\{p(\mathbf{y} \mid \mathbf{x}, \boldsymbol{\theta})\}$; In the Bayesian approach, we also assign specific prior probabilities $p(\xi, \theta)$ to all unknown parameters. According to Baye's rule, we apply the joint MAP estimation to get $(\hat{\mathbf{x}}, \hat{\theta})_{J M A P}=\arg \max _{\mathbf{x}, \theta}\{\ln p(\mathbf{x}, \theta \mid \mathbf{y})\} \propto \arg \min _{\mathbf{x}, \theta}\{-\ln p(\mathbf{y} \mid \mathbf{x}, \theta)-\ln p(\mathbf{x})-\ln p(\theta)\}$. We can see that joint MAP exploit the priors of the unknowns to regularize the ML estimation. Compared to conventional regularization methods [3, 4], the joint MAP has the advantage of an adaptive estimation of the regularization parameter. However in joint MAP, $\ln p(\mathbf{x}, \theta \mid \mathbf{y})$ cannot be obtained analytically for the present problem, and JMAP usually requires a nonlinear optimization with respect to $(\mathbf{x}, \theta)$. Moreover, both $\mathrm{ML}$ and joint MAP are the point estimators which can hardly consider the estimation precision.

The above difficulties of joint MAP can be overcome by the VBA [7, 8] estimation, in which, posterior $p(\mathbf{x}, \theta \mid \mathbf{y})$ is approximated by a family of basic and easily handled probability distributions $q(\mathbf{x}, \theta)$, namely $p(\mathbf{x}, \theta \mid \mathbf{y}) \approx q(\mathbf{x}, \theta)$; and proper $q(\mathbf{x}, \theta)$ are estimated by maximizing variational bound $\mathfrak{L}(\mathbf{x}, \theta)$ as: $\hat{q}(\mathbf{x}, \theta)=\arg _{\max }(\mathbf{x}, \theta)\{\mathfrak{L}(\mathbf{x}, \theta)\}$, where $\mathfrak{L}(\mathbf{x}, \theta)=\int q(\mathbf{x}, \theta) \ln \frac{p(\mathbf{y}, \mathbf{x}, \theta)}{q(\mathbf{x}, \theta)} d \theta d \mathbf{x}$. Generally, $(\mathbf{x}, \theta)$ are supposed to be mutually independent: $q(\mathbf{x}, \theta)=q_{1}(\mathbf{x}) \prod_{i} q_{2}\left(\theta_{i}\right)$. Then $\mathfrak{L}(\mathbf{x}, \theta)$ is maximized by the mean field approximation, for example, $\hat{q}_{2}\left(\theta_{i}\right)=\frac{\exp \left[I\left(\mathbf{x}, \theta_{i}\right)\right]}{\int \exp \left[I\left(\mathbf{x}, \theta_{i}\right)\right] d \theta_{i}}$, where $I(\cdot)$ denotes the partition function, defined as $I\left(\mathbf{x}, \theta_{i}\right)=<\ln p(\mathbf{y}, \mathbf{x}, \boldsymbol{\theta})>_{q_{2}\left(\theta_{-i}\right)}=\int q_{2}\left(\theta_{-i}\right) \ln p(\mathbf{y}, \mathbf{x}, \boldsymbol{\theta}) d \theta_{-i}$, where $\theta_{-i}$ denote the parameter vector except item $\theta_{i}$. In fact, $I\left(\mathbf{x}, \theta_{i}\right)$ can hardly be analytically computed, since it depends on $q_{2}\left(\theta_{-i}\right)$. But VBA inference can still obtain the approximating posterior $\hat{q}(\theta)$ owing to the conjugate priors: $\hat{q}_{2}(\theta)$ should come from the same family of the prior $p(\theta)$ based on the proper combination [11] of the likelihood and conjugate priors.

\section{Heavy tail prior for colored background noise}
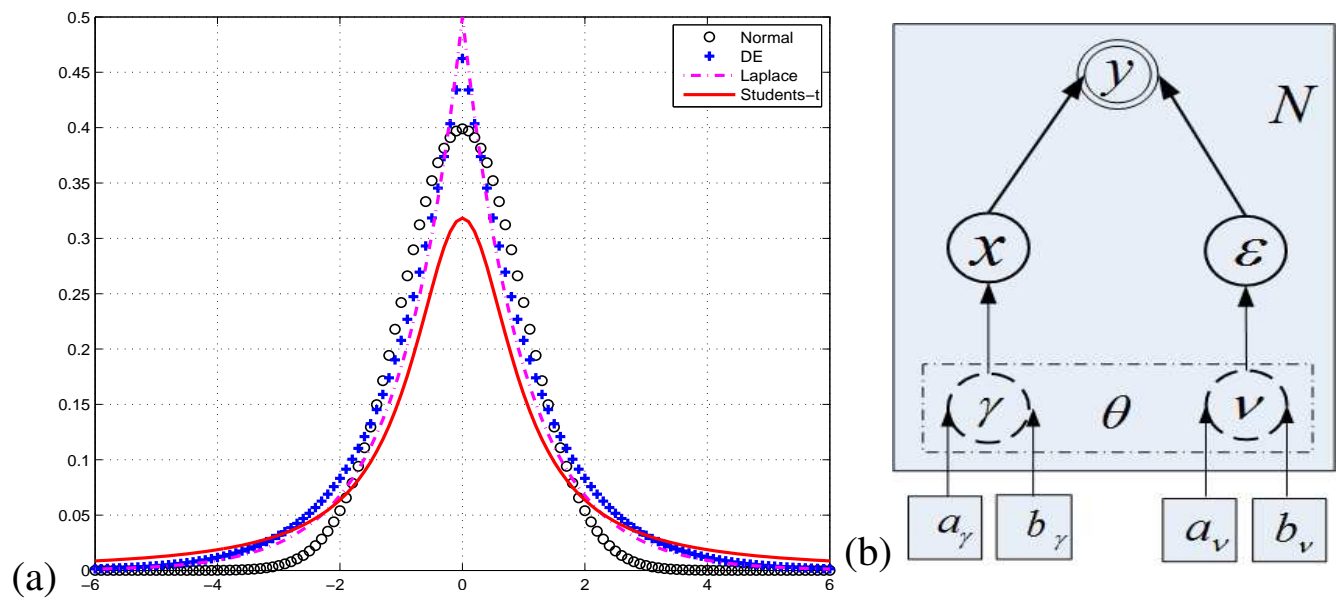

FIGURE 1. (a) Sparse priors on Gaussian normal, Laplace, DE and Student-t; (b) N dimension hierarchical Bayesian Graphical model; Double circle: observed data; Single: unknown variables; Dash: hidden variables; Square: hyper-parameters; Arrow: dependence. 
In wind tunnel experiments, we model the colored noise $\xi$ by the Student-t prior distribution $\operatorname{St}(\xi)$ which has a long heavy tail, rather than the Gaussian distortions whose thin tail excessively penalizes large errors of forward model. Another attractive superposition property is that $S t(\xi)$ can be generated by marginalizing a hidden variable $v$ as $S t(\xi)=\int p(\xi \mid v) p(v) d v$, in which, the conditional prior $p(\xi \mid v)=$ $\mathscr{N}\left(\xi \mid 0, \Sigma_{\xi}^{-1}\right)$ is the multivariate Gaussian distribution, with $\Sigma_{\xi}=\operatorname{Diag}\{v\} \in \mathbb{R}^{N \times N}$ being noise covariance matrix; $\operatorname{Diag}(\cdot)$ denote diagonal matrix; $v=\left\{v_{n}\right\}_{N} \in \mathbb{R}^{N \times 1}$ denote the noise precision vector; and the hyper-prior $p(v)$ is the Gamma distribution: $p(v)=\prod_{n=1}^{N} \mathscr{G}\left(v_{n} \mid a_{v}, b_{v}\right)=\prod_{n=1}^{N} \Gamma\left(a_{v}\right)^{-1}\left(b_{v}\right)^{a_{v}} v_{n}^{a_{v}-1} e^{-b_{v} v_{n}}$, with $a_{v}, b_{v}$ being the hyper-parameters of $p(v)$, and $\Gamma(x)=\int t^{x-1} e^{-t} d t$.

According to proposed forward model of (1), the likelihood $p(\mathbf{y} \mid \mathbf{x}, v)$ is determined by the conditional prior $p(\xi \mid v)=\mathscr{N}\left(\xi \mid 0, \Sigma_{\xi}^{-1}\right)$ as:

$$
p(\mathbf{y} \mid \mathbf{x}, v)=\frac{\left|\Sigma_{\xi}\right|^{1 / 2}}{(2 \pi)^{N / 2}} e^{-\frac{1}{2}(\mathbf{y}-\mathbf{C x})^{H} \Sigma_{\xi}(\mathbf{y}-\mathbf{C x})},
$$

where operator $(\cdot)^{H}$ denotes conjugate transpose.

\section{Sparse prior on acoustic power image}

Acoustic source in wind tunnel experiments are generated by the interaction of the air flow with special parts of the vehicle surface. Therefore, sources are located on some particular locations, while on the other parts, there is nearly no emission. This is why the acoustic power $\mathbf{x}$ becomes a K-sparsity signal when the source plane is discretized into $\mathrm{N}$ patches. Such a sparse distribution can be represented by the distribution that has a high probability density around zero (sparsity) and a long heavy tail (dynamic range).

Here we apply the Student-t prior $S t(\mathbf{x})$ [7] to enforce the sparsity and wide dynamic range of source power distribution. Owing to the superposition property of Student$\mathrm{t}$ prior, the hidden variable $\gamma$ is marginalized out for $\operatorname{St}(\mathbf{x})=\int p(\mathbf{x} \mid \gamma) p(\gamma) d \gamma$, where $p(\mathbf{x} \mid \gamma)=\mathscr{N}\left(\mathbf{x} \mid 0, \Sigma_{x}^{-1}\right)$ is assigned to multivariate Gaussian distribution, in which, $\Sigma_{x}$ denote power covariance matrix, defined as $\Sigma_{x}=\operatorname{Diag}\{\gamma\} \in \mathbb{R}^{N \times N}$ with $\gamma=\left\{\gamma_{n}\right\}_{N} \in$

$\mathbb{R}^{N \times 1}$ being the power precision vector; and $p(\gamma)=\prod_{n=1}^{N} \mathscr{G}\left(\gamma_{n} \mid a_{\gamma}, b_{\gamma}\right)$, where $a_{\gamma}, b_{\gamma}$ denote the hyper-parameters of $p(\gamma) .0<\gamma_{n}<1$ greatly promotes the sparsity as shown by the solid curve in Fig.1a; while for $\gamma_{n} \rightarrow \infty S t\left(x_{n}\right)$ approaches a Gaussian normal distribution as shown by the circle curve. Compared to the Double Exponential (DE) prior as shown by the dot curve, St $\left(x_{n}\right)$ can have different $\gamma_{n}$ for each $x_{n}$, while the DE prior requires precisely only two parameters for all $\mathbf{x}$ in order to achieve a similar sparsity and heavy tail distribution. In addition, the Laplacian distribution is one of the DE cases as the dash curve shown. 


\section{VBA parameter estimations}

In Fig.1a, the graphical model [11] describes the dependencies between the observed data $\mathbf{y}$, all of the unknown variables $\mathbf{x}$, as well as the hidden variables $\theta=[\gamma, v]^{T}$ and the initialized hyper-parameters $\phi_{0}=\left[a_{\gamma}^{0}, b_{\gamma}^{0}, a_{v}^{0}, b_{v}^{0}\right]^{T}$. According to Bayes' rule, we have $p\left(\mathbf{x}, \theta \mid \boldsymbol{y}, \phi_{0}\right) \propto p\left(\boldsymbol{y} \mid \mathbf{x}, \boldsymbol{\theta}, \phi_{0}\right) p\left(\mathbf{x} \mid \boldsymbol{\theta}, \phi_{0}\right) p\left(\boldsymbol{\theta} \mid \phi_{0}\right)$. According to the multivariate Gaussian likelihood in (2) and the superpositions of Student-t priors on $\mathbf{x}$ and $\xi$, we can write the posterior as

$$
\begin{aligned}
p\left(\mathbf{x}, \theta \mid \boldsymbol{y}, \phi_{0}\right) & =\mathscr{N}\left(\mathbf{x} \mid \boldsymbol{y}-\mathbf{C} \mathbf{x}, \Sigma_{\xi}^{-1}\right) \mathscr{N}\left(\mathbf{x} \mid 0, \Sigma_{x}^{-1}\right) \\
& \mathscr{G}\left(\gamma \mid a_{\gamma}^{0}, b_{\gamma}^{0}\right) \mathscr{N}\left(\xi \mid 0, \Sigma_{\xi}^{-1}\right) \mathscr{G}\left(v^{0} \mid a_{v}, b_{v}^{0}\right)
\end{aligned} .
$$

Due to the conjugate prior, the approximating posterior belongs to the Student-t distribution which is expressed by the multivariate Gaussian distribution $\hat{q}(\mathbf{x})$ and Gamma distribution $\hat{q}(\gamma)$, similarly for $\hat{q}(v)$ as follows:

$$
\left\{\begin{array}{l}
\hat{q}(\mathbf{x})=\mathscr{N}\left(\mathbf{x} \mid \hat{\mu}_{x}, \hat{\Sigma}_{x}\right) \\
\hat{q}(\gamma)=\prod_{n=1}^{N} \mathscr{G}\left(\gamma_{n} \mid \hat{a}_{\gamma}, \hat{b}_{\gamma}^{n}\right) \\
\hat{q}(v)=\prod_{n=1}^{N} \mathscr{G}\left(v_{n} \mid \hat{a}_{v}, \hat{b}_{v}^{n}\right)
\end{array}\right.
$$

where the averaged image $\hat{\mu}_{x}$ is our final goal; the covariance matrix $\hat{\Sigma}_{x}$ offers the estimation precision by the VBA approach; and the expected variable estimates are:

$$
\left\{\begin{array}{l}
\hat{\mu}_{x}=\hat{\Sigma}_{x} \mathbf{C}^{T}<\Sigma_{\xi}>\boldsymbol{y} \\
\hat{\Sigma}_{x}=\left(\mathbf{C}^{T}<\Sigma_{\xi}>\mathbf{C}+<\Sigma_{x}>\right)^{-1} \\
\hat{a}_{\gamma}=a_{\gamma}^{0}+\frac{N}{2}, \quad \hat{b}_{\gamma}^{n}=b_{\gamma}^{0}+\frac{1}{2}<\mathbf{x x}^{T}>_{n n} \\
\hat{a}_{v}=a_{v}^{0}+\frac{N}{2}, \quad \hat{b}_{v}^{n}=b_{v}^{0}+\frac{1}{2}<\xi \xi^{T}>_{n n}
\end{array}\right.
$$

where the operator $(\cdot)_{n n}$ denotes the $n$th diagonal item, and $\langle\cdot\rangle$ denotes expectation, which is calculated as:

$$
\left\{\begin{array}{l}
<\Sigma_{\xi}>=\operatorname{Diag}\left\{<v_{n}>\right\}_{N}=\operatorname{Diag}\left\{<\hat{a}_{v} / \hat{b}_{v}^{n}>\right\}_{N} \\
<\Sigma_{x}>=\operatorname{Diag}\left\{<\gamma_{n}>\right\}_{N}=\operatorname{Diag}\left\{<\hat{a}_{\gamma} / \hat{b}_{\gamma}^{n}>\right\}_{N} \\
<\mathbf{x x}^{T}>=\hat{\mu}_{x} \hat{\mu}_{x}^{T}+\hat{\Sigma}_{x} \\
<\xi \xi^{T}>=\boldsymbol{y} \boldsymbol{y}^{T}-2 \mathbf{C} \hat{\mu}_{x} \boldsymbol{y}^{T}+\mathbf{C}<\mathbf{x} \mathbf{x}^{T}>\mathbf{C}^{T}
\end{array}\right.
$$

More details of above calculations can be found in literature [11, 7, 8].

\section{Computational analysis}

From the solutions in (5), $\hat{\Sigma}_{x}$ involves the matrix inversion which cannot be calculated explicitly. We suggest to approximate $\hat{\Sigma}_{x}$ with a circulant matrix as $\hat{\Sigma}_{x} \approx(<\bar{v}\rangle$ $\left.\mathbf{C}^{H} \mathbf{C}+<\bar{\gamma}>\right)^{-1}$, where $\bar{v}=\sum_{n=1}^{N} v_{n}, \bar{\gamma}=\sum_{n=1}^{N} \gamma_{n}$ denote the arithmetic mean. Then the products of circulant matrices can be efficiently computed in the Discrete Fourier Transform (DFT) domain. In (5), the estimated expectation $\hat{\mu}_{x}$ of source powers can 
be analytically expressed as $\hat{\Sigma}_{x}^{-1} \hat{\mu}_{x}=\mathbf{C}^{H}<\Sigma_{\xi}>\mathbf{y}$. This linear system of equations is solved iteratively with a conjugate gradient algorithm, which requires $O(N \log N)$ computations per iteration to solve for a solution vector $\mathbf{x}$ of size $N$. If $Q$ iterations are needed, total computations are of $O(Q N \log N)$, which remains moderate.

\section{SIMULATIONS}

The simulation configuration is based on the wind tunnel experiments in Fig.3a: the distance between sensor and source plane is $4.50 \mathrm{~m}$; and there are $M=64$ sensors; The source plane is discretized into $5 \mathrm{~cm} \times 5 \mathrm{~cm}$ grids. In Fig. $2 \mathrm{a}$, source powers $\mathbf{x}$ are generated by 4 monopoles and 5 extended sources with $14 \mathrm{~dB}$ dynamic range, and the image size is of $27 \times 17$ pixels. The colored noise is generated by using the Gaussian white noise filtered by a low pass filter (cut-off frequency $3000 \mathrm{~Hz}$ ), and the averaged Signal-to-Noise Ratio (SNR) is set to $0 \mathrm{~dB}$.

(a)

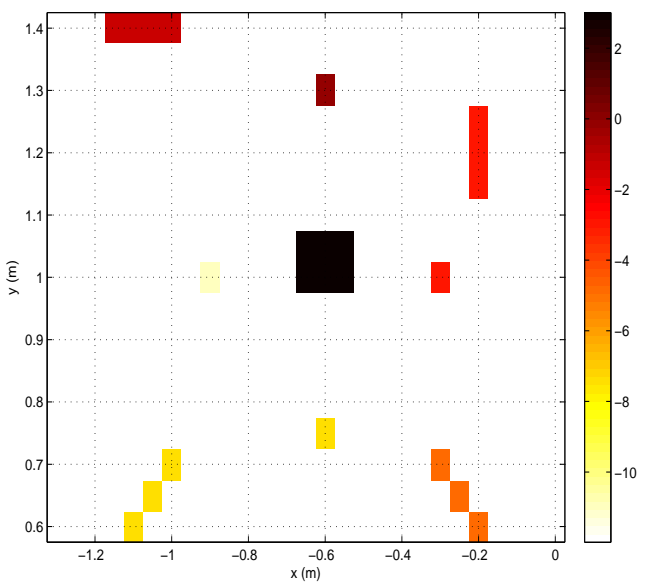

(c)

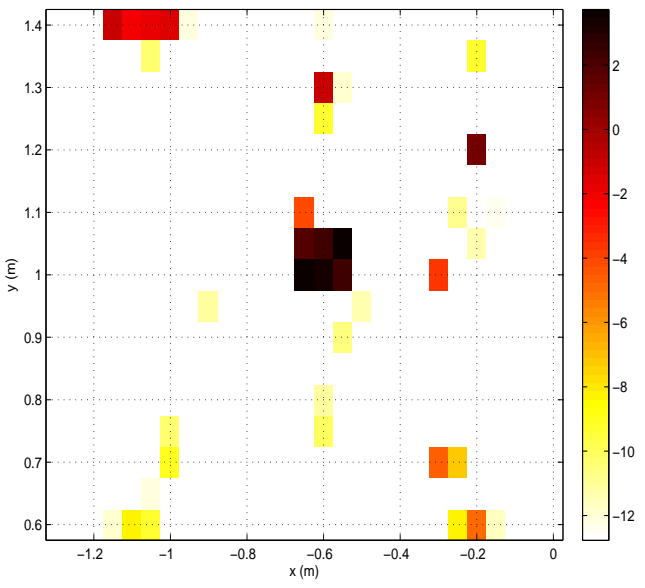

(b)

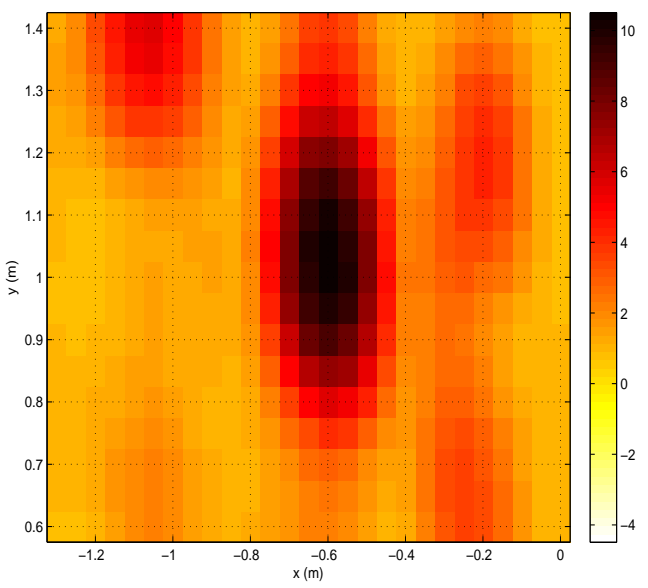

$(d)$

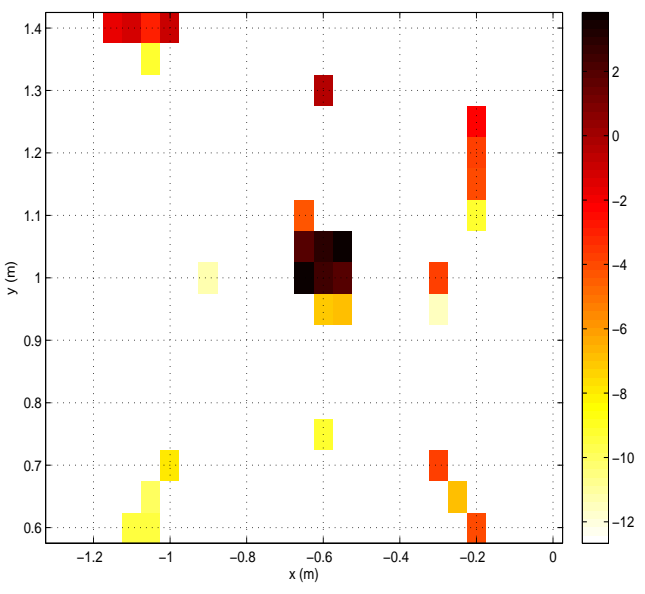

FIGURE 2. Simulation at 2500Hz, OdB SNR under colored noise, 14dB span: (a) Source power distribution (b) Beamforming power (c) Bayesian joint MAP and (d) Proposed VBA inversion

In Fig.2b-d, the beamforming merely gives the strong sources and failed to distinguish most of the weak sources; our proposed VBA inference outperforms the joint MAP 
method [9] due to its more precise localization and power estimation, especially for the better noise suppression.

\section{WIND TUNNEL EXPERIMENTS}

Wind tunnel experiments are designed to reconstruct the positions and acoustic powers on the traveling car surface. The grid is of $5 \mathrm{~cm}$, and source plane is thus of $31 \times 101$ pixels. The wind speed is $160 \mathrm{~km} / h$; there are 524288 samplings with the sampling frequency $f_{s}=2.56 \times 10^{4} \mathrm{~Hz}$. The total samplings are separated into $\mathrm{I}=204$ blocks with 2560 samplings in each bloc. The working frequency is $2500 \mathrm{~Hz}$ which is within the frequency range of the human acoustic perception. The image results are obtained in frequency domain shown by normalized $\mathrm{dB}$ images with $10 \mathrm{~dB}$ span. The propagation matrix $\mathbf{C}$ in (1) is rectified for the wind refraction and ground reflection as discussed in [9].

(a)

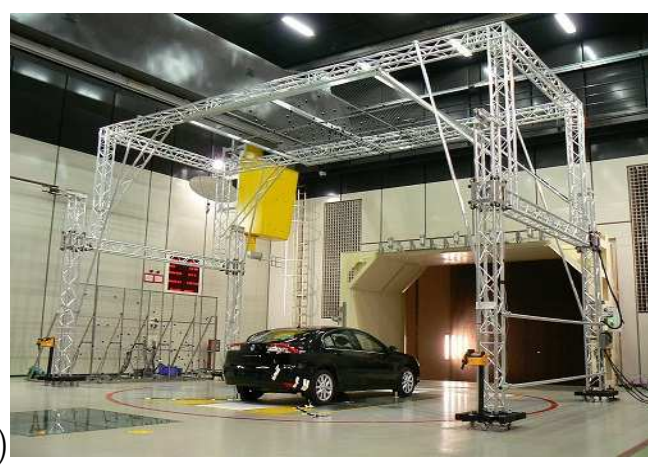

(c)

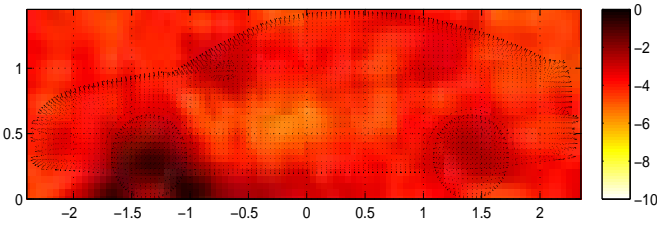

$(e)$

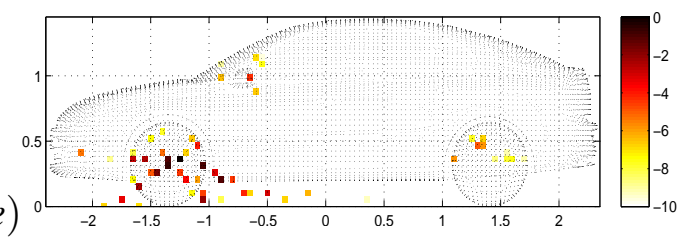

(b)
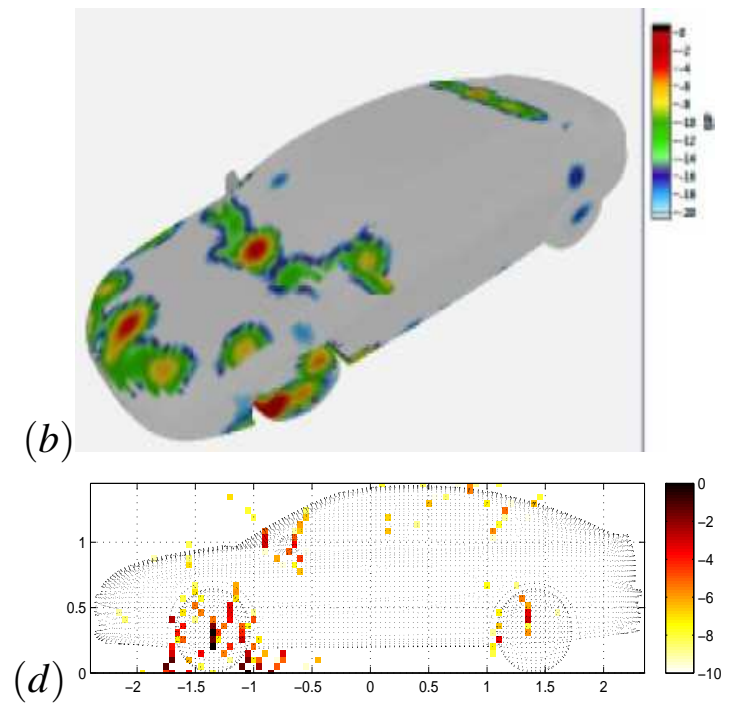

$(f)$

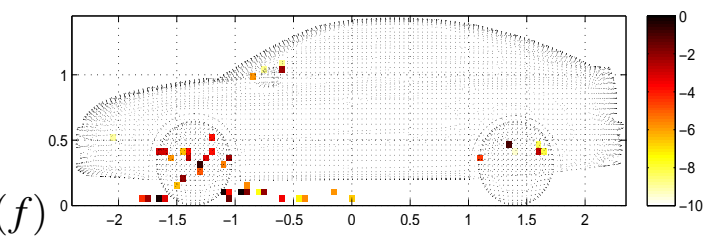

FIGURE 3. Vehicle acoustic imaging at 2500Hz: (a) Wind tunnel S2A Renault France [12](b) Acoustic imaging [12] (c) Beamforming (d) DAMAS (e) joint MAP and (f) Proposed VBA.

Fig. 3 illustrates the Wind Tunnel S2A configuration, a previous result offered by Renault company researchers, as well as the estimated powers of mentioned methods. Due to the high side-lobe effect, beamforming merely gives a fuzzy image of strong sources in Fig.3c; DAMAS well deconvolves the beamforming image and discovers sources around the wheels and rear-view mirror, however, many false targets are also detected on the air in Fig.3d; In Fig.3e and f, the joint MAP inference via DE prior [9] and proposed VBA inference not only manages to distinguish the strong sources around the two wheels and rear-view mirror, but also successfully reconstructs the weak ones 
on the front cover and light. Furthermore, the background noise suppression in proposed VBA are much better achieved than others owing to Student-t prior.

\section{CONCLUSION}

We proposed a VBA inference via Student-t priors on the source power and colored noise for super spatial resolution, wide dynamic range and robust parameter estimation. On simulations and real data in wind tunnel, the proposed VBA approach has been validated by comparison to classical methods. However, the VBA still requires a huge amount of computation in calculating $\hat{\Sigma}_{x} \approx\left(<\bar{v}>\mathbf{C}^{H} \mathbf{C}+<\bar{\gamma}>\right)^{-1}$. For real-time processing, it needs to employ the Graphical Processing Unit (GPU) for hardware accelerations.

\section{ACKNOWLEDGMENTS}

We greatly thank Renault SAS for offering real data, and appreciate valuable comments from Prof. Udo von Toussaint of Max-Planck-Institut Germany, Dr. Frédérique Barbaresco of Thales France, as well as Prof. HUANG Xiaotao of NUDT University China.

\section{REFERENCES}

1. J. Chen, K. Yao, and R. Hudson, Source localization and beamforming, Signal Processing Magazine, IEEE 19, 30-39 (2002).

2. T. Brooks, and W. Humphreys, A Deconvolution Approach for the Mapping of Acoustic Sources (DAMAS) determined from phased microphone arrays, Journal of Sound and Vibration 294, 856879 (2006), ISSN 0022-460X.

3. T. Yardibi, J. Li, P. Stoica, N. S. Zawodny, and L. N. Cattafesta,A covariance fitting approach for correlated acoustic source mapping, Journal of The Acoustical Society of America 127, 2920-31 (2010)

4. N. Chu, J. Picheral, A. Mohammad-Djafari, and N. Gac, A robust super-resolution approach with sparsity constraint in acoustic imaging, Applied Acoustics 76, 197-208 (2014).

5. G. Oliveri, P. Rocca, and A. Massa, A Bayesian-Compressive-Sampling-Based Inversion for Imaging Sparse Scatterers, Geoscience and Remote Sensing, IEEE Transactions on 49, 3993-4006 (2011).

6. J. Antoni, A Bayesian approach to sound source reconstruction: optimal basis, regularization, and focusing, The Journal of the Acoustical Society of America 131, 2873-2890 (2012).

7. D. Tzikas, A. Likas, and N. Galatsanos, Variational Bayesian sparse kernel-based blind image deconvolution with Student's-t priors, IEEE Transactions on Image Processing 18, 753-764 (2009).

8. A. Mohammad-Djafari, Bayesian approach with prior models which enforce sparsity in signal and image processing, EURASIP Journal on Advances in Signal Processing 2012, 52 (2012), ISSN 16876180.

9. N. Chu, A. Mohammad-Djafari, and J. Picheral, Robust Bayesian super-resolution approach via sparsity enforcing a priori for near-field aeroacoustic source imaging, Journal of Sound and Vibration 332, 4369-4389 (2013), ISSN 0022-460X.

10. A. Massa, and G. Oliveri, Bayesian compressive sampling for pattern synthesis with maximally sparse non-uniform linear arrays, IEEE Transactions on Antennas and Propagation 59, 467-681 (2011).

11. M. Jordan, Z. Ghahramani, T. Jaakkola, and L. Saul, An introduction to variational methods for graphical models, Machine learning 37, 183-233 (1999).

12. A. Menoret, N. Gorilliot, and J.-L. Adam, Acoustic imaging in wind tunnel S2A, "Acoustic imaging in wind tunnel S2A," in 10th Acoustics conference (ACOUSTICS2010), Lyon, France, 2010. 